\title{
Chiral effects in normal and superconducting carbon nanotube-based nanostructures
}

\author{
A.V. Parafilo ${ }^{1}$, I.V. Krive ${ }^{1,2,3}$, E.N. Bogachek ${ }^{4}$, U. Landman ${ }^{4}$, \\ R.I. Shekhter ${ }^{2}$, and M. Jonson ${ }^{2,5,6}$ \\ ${ }^{1}$ B. Verkin Institute for Low Temperature Physics and Engineering of the National Academy of Sciences of Ukraine \\ 47 Lenin Ave., Kharkov 61103, Ukraine \\ E-mail: krive@ilt.kharkov.ua \\ ${ }^{2}$ Department of Physics, University of Gothenburg, Göteborg SE-412 96, Sweden \\ ${ }^{3}$ Physical Department, V.N. Karazin National University, Kharkov 61077, Ukraine \\ ${ }^{4}$ School of Physics, Georgia Institute of Technology, Atlanta, Georgia 30332-0430, USA \\ ${ }^{5}$ School of Engineering and Physical Sciences, Heriot-Watt University, Edinburgh EH14 4AS, Scotland, UK \\ ${ }^{6}$ Division of Quantum Phases and Devices, School of Physics, Konkuk University, Seoul 143-701, Korea
}

Received May 6, 2010

\begin{abstract}
The novel phenomenon of chiral tunneling in metallic single-wall carbon nanotubes is considered. It is induced by the interplay of electrostatic and pseudomagnetic effects in electron scattering in chiral nanotubes and is characterized by the oscillatory dependence of the electron transmission probability on nanotube chiral angle and the strength of the scattering potential. The appearance of a special (Aharonov-Bohm-like) phase in chiral tunneling affects various phase-coherent phenomena in nanostructures. We considered chiral effects in: (i) the persistent current in a circular nanotube, (ii) the Josephson current in a nanotube-based SNS junction, and (iii) resonant electron tunneling through a chiral nanotube-based quantum dot.
\end{abstract}

PACS: 73.23Ra Persistent currents;

72.10.-d Theory of electronic transport; scattering mechanisms;

74.50.+r Tunneling phenomena; Josephson effects.

Keywords: chiral tunneling, persistent currents, Josephson current, carbon nanotube.

\section{Introduction}

One of the most spectacular phenomena in physics is the Aharonov-Bohm (AB) effect predicted in 1959 [1] and a year later realized in experiment [2] (see also the review [3] and references therein). This effect is of fundamental nature, it has a simple theoretical formulation and numerous theoretical and experimental applications. The last assertion concerns first of all condensed matter physics where the $\mathrm{AB}$ effect is a key idea in a vast amount of theoretical and experimental papers.

One of the first among the most significant papers on the $\mathrm{AB}$ effect in condensed matter physics is the work by I.O. Kulik on non-decaying electric currents in normal metal systems published in 1970 [4]. In this paper it was predicted that a perfect (impurity-free) small metallic cylinder threaded by magnetic field will support a non- dissipative (persistent) electric current with the periodicity of a single-flux quantum $\Phi_{0}=h c / e$ (see also $[5,6]$ ) and an amplitude (at low temperatures) given by the singleelectron current $\sim e v_{F} / L$ times the number of transverse channels for a few-channel ring (here $v_{F}$ is the Fermi velocity and $L$ is the ring circumference). At that time 15 years before the advent of mesoscopic physics - the prediction that certain physical characteristics of a real many body (macroscopic) system (now it is better to say - mesoscopic) could be sensitive to a single-electron contribution sounded bizarre for many physicists. Although the fundamental nature of Kulik's prediction was evident (the paper was published in the most prestigeous physics journal in the Soviet Union), prospects for the experimental observation of this effect looked obscure. Nevertheless, rather soon the prediction was confirmed, at first in indirect experiments $[7,8]$ with massive cylinders where the 
$\mathrm{AB}$ persistent currents were induced by electronic states localized near the surface (whispering gallery states) and forming effectively a doubly connected (ring) geometry $[9,10]$. Later, in the beginning of the 1990's, persistent currents were measured in a single metallic (gold) ring [11] (diffusive regime of electron transport [12]) and soon in a quantum ring formed in 2D electron gas (EG) [13]. In 2DEG electron transport is ballistic and the measurements [13] were in good agreement with Kulik's prediction [4].

Since that time persistent currents were always a hot topic in condensed matter physics and there is a vast literature on the problem (see e.g. [14] and the reviews [15-17]). Aharonov-Bohm oscillations were observed not only in metallic rings and cylinders, but in more exotic systems like, e.g., conducting quasi-1D materials with charge density wave excitations [18], where the $\mathrm{AB}$ effect is induced by the quantum coherent dynamics of collective modes $[19,20]$. Theoretical studies of nontraditional AB effects in condensed matter range from the calculation of persistent currents in dielectrics [21] to the study of $\mathrm{AB}$ oscillations induced by superconducting plasmons [22].

Our purpose here is to consider persistent currents in circular carbon nanotubes and supercurrents in nanotubebased superconductor/normal metal/superconductor (SNS) junctions. Both structures have been studied experimenttally. The ring-shaped nanotubes (including rings of singlewall nanotubes) were observed and investigated in Refs. 23, 24 while measurements of the Josephson current in a single-wall nanotube-based SNS junction was reported in Ref. 25.

What is specific in the transport properties of carbon nanotube-based mesoscopic structures as compared to the «ordinary» metallic nanowires? Electron transport in metallic single-wall nanotubes (SWNT) is ballistic and this property is explained by a specific scattering of charge carriers by the nanotube defects (see, e.g., [26]). Conduction electrons in SWNT are Dirac-like particles and their relativistic spectrum leads to certain peculiarities in electron scattering. In particular long-range electrostatic potentials in metallic nanotubes do not scatter electrons at all. This effect is explained by the conservation of helicity for relativistic particles. In quantum field theory the phenomenon of particle free penetration through potential barriers is known as the Klein paradox (see the discussion in Ref. 27). The specific features of electron scattering in chiral nanotubes and their influence on persistent and super-currents in carbon nanotube-based devices is the goal of the present paper.

In Sec. 2 we introduce the new concept of chiral tunneling in metallic SWNTs. For a special $2 \times 2$-matrix scattering potential the transmission and reflection amplitudes are derived. It is shown that in the local limit the transmission coefficient $D(\theta)$ is an oscillating function of the chiral phase $\varphi_{c}=U_{0} \cos \theta$, where $U_{0}$ is the dimensionless strength of the scattering potential and $\theta$ is the nanotube chiral angle. Resonant chiral tunneling, $D_{r}(\theta)=0$, occurs for quantized values of the chiral phase $\varphi_{c}=\pi n$ (where $n$ is an integer).

In Sec. 3 we evaluate the persistent current in a circular metallic SWNT in the presence of chiral tunneling. We show that the chiral phase $\varphi_{c}$ plays a crucial role in the magnetic response of circular carbon nanotubes. In particular, the parity of the chiral resonance (even or odd $n$ ) determines the character of the magnetic response (paramagnetic or diamagnetic persistent current). The existance of non-equilibrium spontaneous persistent currents in an isolated nanotube ring with asymmetric populations of the $\pm k_{F}$-valleys is briefly discussed.

In the next section we consider the influence of chiral effects on the supercurrent in a SWNT-based SNS junction. The equation for the bound state energies (AndreevKulik levels) in the presence of chiral tunneling is derived. It is shown that for energy independent phase factors (forward and backward scattering phases and the chiral phase) the spectral equation expressed in terms of scattering data coincides with the corresponding equation for standard SNS junction. All information specific to chiral tunneling is hidden in the oscillatory dependence of the junction transparency on the chiral angle and chiral phase. In particular we discuss here the interesting possibility to fabricate highly transparent junction by using high quality carbon nanotubes with small chiral angles.

In Sec. 5 the resonant electron transport through a «chiral» quantum dot (QD) (i.e., the QD based on a chiral metallic SWNT) is considered. We show that in the presence of a chiral scatterer inside the tube the spacings between the resonant conductance peaks (measured by varying the gate voltage) strongly depend on the nanotube chiral angle and the chiral phase (which in principle can be considered as a controllable parameter). The distribution of the number of conductance maxima on the level spacing ranges from $\delta$-function like peaks for armchair nanotubes (equidistant spectrum of QD energy levels) to a smooth Wigner-Dysonlike distribution (quasi-random energy spectrum) in chiral nanotubes in the limit of weak chiral tunneling $(D \ll 1)$.

In the Conclusion we summarize the main results and briefly discuss the influence of electron-electron interaction on chiral tunneling.

\section{Chiral tunneling}

We evaluate the transmission probability for electron scattering by special defects (see below) in carbon nanotubes. We will assume that the defect potentials are longranged and do not induce inter-valley ( $\delta k \ll 2 k_{F}$ ) electron scattering. Thus in our model the metallic SWNT Hamiltonian is diagonal in the valley index $j= \pm$ and takes the form [28]

$$
H_{ \pm}= \pm \hbar v_{F}\left(\begin{array}{cc}
0 & \exp ( \pm i \theta) \hat{p}_{x} \\
\exp (\mp i \theta) \hat{p}_{x} & 0
\end{array}\right) .
$$


Here $v_{F}$ is the Fermi velocity, $\hat{p}_{x}=-i \partial_{x}, \theta$ is the chiral angle of the nanotube $(0 \leq \theta \leq \pi / 6)$ and the $x$-axis is directed along the cylinder axis. Notice that we follow [28] in the definition of chiral angle $(\theta=0$ for the armchair nanotube and $\theta=\pi / 6$ for the zigzag nanotube) which is different from the definition used in Refs. 29,30. The presence of chiral factors $\exp ( \pm \theta)$ in the Hamiltonian Eq. (1) results in special scattering of electrons by a non-diagonal potential [28] — the effect of which we in what follows will call chiral tunneling.

The electrostatic (scalar) potential is diagonal in the pseudospin indices and can not induce electron backscattering in our model Eq. (1) due to the conservation of helicity for massless Dirac particles (the Klein paradox). To get nontrivial scattering of chiral particles we consider the matrix potential

$$
\hat{V}_{S}(x)=\left(\begin{array}{ll}
V(x) & V(x) \\
V(x) & V(x)
\end{array}\right)
$$

which mixes the sublattice components of the electron wave function. For simplicity we consider all matrix elements to be real and equal. An effective scattering potential of the form of Eq. (2) was suggested in Ref. 28 for the description of electron scattering in metallic carbon nanopeapods. It is induced by the hybridization of fullerene molecular orbitals (LUMO) with the conduction electron states in the nanotube.

To proceed further we will consider the scattering potential Eq. (2) to be «local». However, we can not take the spatial dependence of $V_{S}(x)$ to be simply $\delta(x)$. This is because a $\delta$-function scattering potential is ill-defined in the context of the 1D Dirac equation. One has additionally to define the value of the fermion wave function at the singular point $x=0$ (the wave function has a jump at this point). In order to correctly solve the problem, we at first consider a rectangular potential of width $a$ and height $V_{0}$, which allows us to get an analytical solution for the scattering problem. Then we consider the local scatterer limit by letting $a \rightarrow 0, \quad V_{0} \rightarrow \infty$ while keeping the product $V_{0} a$ constant.

The transmission and reflection amplitudes for the rectangular potential is found by matching the plane wave and evanescent mode solutions of the Dirac equation at the points $x=0, a$. The transmission amplitude, for instance, takes the form

$$
t(E)=\frac{1}{2 i \kappa \hbar v_{F}} \frac{\exp \{i[2 \theta-a(k+q)]\}\left(V_{0}-E\right)}{\left[\left(V_{0}-E\right)^{2}+\hbar^{2} v_{F}^{2}\left(\kappa^{2}-q^{2}\right)+V_{0} \mathrm{e}^{i \theta}\left(2 q \hbar v_{F}-V_{0} \mathrm{e}^{i \theta} \sin a \kappa\right)\right] \sin a \kappa-2 i \hbar v_{F}\left(V_{0}-E\right) \kappa \cos a \kappa},
$$

where $\kappa=\sqrt{V_{0}^{2} \cos ^{2} \theta-2 E V_{0}+E^{2}} / \hbar v_{F}, q=V_{0} \cos \theta / \hbar v_{F}$, $k=E / \hbar v_{F}$.

In the limit we are interested in the Eq. (3) is strongly simplified. The corresponding transmission and reflection amplitudes are

$$
\begin{aligned}
& t=\frac{\cos \theta \exp \left(-i U_{0} \cos \theta\right)}{\cos \theta \cos \left(U_{0} \cos \theta\right)+i \sin \left(U_{0} \cos \theta\right)}, \\
& r=-\frac{\sin \theta \sin \left(U_{0} \cos \theta\right)}{\cos \theta \cos \left(U_{0} \cos \theta\right)+i \sin \left(U_{0} \cos \theta\right)} .
\end{aligned}
$$

From Eq. (4) we get expressions for the scattering data which will be used in what follows (i.e., for the transmission coefficient $D=t t^{*}$, and the forward, $\delta_{f}$, and backward, $\delta_{b}$, scattering phases)

$$
\begin{gathered}
D(\theta)=\frac{\cos ^{2} \theta}{\cos ^{2}\left(U_{0} \cos \theta\right) \cos ^{2} \theta+\sin ^{2}\left(U_{0} \cos \theta\right)} \\
R(\theta)=1-D(\theta) ; \\
\delta_{f}(\theta)=\arctan \left[\frac{\sin \left(2 U_{0} \cos \theta\right) \cos ^{2}(\theta / 2)}{\cos ^{2}\left(U_{0} \cos \theta\right) \cos \theta-\sin ^{2}\left(U_{0} \cos \theta\right)}\right]= \\
=U_{0} \cos \theta+\delta_{b}(\theta), \\
\delta_{b}(\theta)=\arctan \left[\frac{\tan \left(U_{0} \cos \theta\right)}{\cos \theta}\right] .
\end{gathered}
$$

Here $U_{0}=a V_{o} / \hbar v_{F}$ is the dimensionless strength of the «local» scattering potential in our model. Notice that the formula for the transmission coefficient $D$ after a change of notations coincides with the analogous expression for the transmission coefficient in graphene [31]. In our case the chiral angle $\theta$ plays the role of the incident angle of a particle scattered by a rectangular barrier in 2D graphene.

To understand why the quantity $U_{0} \cos \theta$ appears in the arguments of some trigonometric functions, it is useful to find the spectrum of the Dirac equation in the constant matrix potential

$$
\hat{V}_{s}=\left(\begin{array}{cc}
V_{d} & V_{o} \\
V_{o}^{*} & V_{d}
\end{array}\right)
$$

From Eqs. (1), (7) we immediately get the spectrum

$$
E=V_{d} \pm \hbar v_{F} \sqrt{\left[p+\tilde{U}_{0} \cos (\theta-\alpha)\right]^{2}+\tilde{U}_{0}^{2} \sin ^{2}(\theta-\alpha)},
$$

where $\tilde{U}_{0} \equiv\left|V_{o}\right| / \hbar v_{F}$ and $V_{o}=\left|V_{o}\right| \exp (i \alpha)$. We see that the only effect of the diagonal potential $V_{d}$ is a constant shift of the energy spectrum. The off-diagonal potential $V_{o}$ - (i) induces a gap (if $\theta \neq \alpha$ ), which mixes left- and rightmoving components of the wave function (i.e. it leads to backscattering events), and (ii) plays the role of «vector» potential by shifting the momentum to $p+U_{o} \cos (\theta-\alpha)$. Notice that the potential $V_{o}$ has to be odd with respect to 

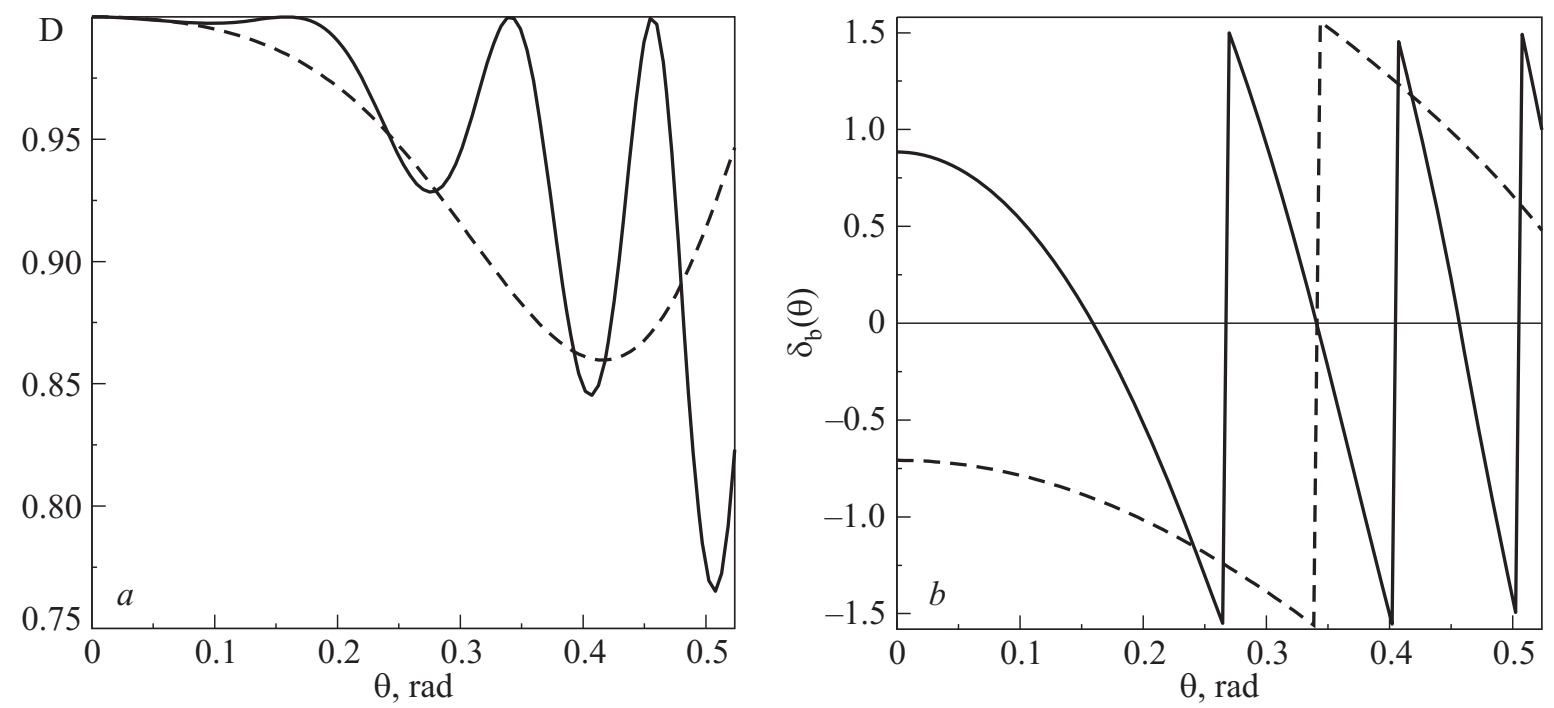

Fig. 1. Transmission coefficient $(a)$ and backward scattering phase $(b)$ as a function of chiral angle at different values of potential strength $U_{0}$ : solid curve corresponds to $U_{0}=70$, dashed curve $-U_{0}=15$.

time reversal $(t \rightarrow-t)$. Both properties are crucial for chiral tunneling. We will see in the next section that the quantity $U_{0} \cos \theta$ (we consider real potentials) changes the Aharonov-Bohm phase in the problem of persistent currents. It will be convenient for as to call $\phi_{c}(\theta)=$ $=\left(U_{0} / 2 \pi\right) \cos \theta$ the dimensionless chiral flux .

It is readily seen from Eqs. (5), (6) that for an armchair $(\theta=0)$ nanotube $D(0)=1$ irrespective of the potential strength, which is a demonstration of the Klein paradox in non-chiral metallic nanotubes. In addition, $D(\theta)=1$ at $U_{0} \cos \theta=\pi N$ ( $N$ is an integer). The minimal value of the transmission probability, $D_{\min }=\cos ^{2} \theta$, is reached at $U_{0} \cos \theta=\pi(N+1 / 2)$. We will refer to these cases as onand off-resonance chiral tunneling. The above considered «quantization conditions» [31] are typical for quantum resonant transport (see, e.g., $[32,33]$ where an analogous formula for the transmission coefficient was obtained for resonant heat transport through a Luttinger liquid constriction). On- and off-resonance conditions for chiral tunneling are analogous to the corresponding conditions of constructive $\left(\Phi / \Phi_{0}=N\right.$, where $\Phi$ is the magnetic flux and $\Phi_{0}=h c / e$ is the flux quantum) and destructive $\left(\Phi / \Phi_{0}=N+1 / 2\right)$ interference for Aharonov-Bohm interferometer (see e.g., Eq. (4.25) of Ref. 34). It is worth to note that unlike in other resonant scattering problems, it is the potential strength (and not the energy of bound states) that is quantized in our case. The dependence of the transmission coefficient and scattering phases on the chiral angle for different values of potential strength is shown in Fig. 1.

\section{Persistent currents in chiral nanotubes}

Several theoretical papers have studied persistent currents in ring-shaped SWNTs. They mostly deal with im- purity free nanotubes and the results obtained concern specific properties of fullerene toroids [35], the differences in magnetic response for metallic and small gap semiconducting nanotubes [36], and the influence of electron-electron interaction on persistent currents in defect-free SWNTs [37]. The presence of a short-range scatterer which induce inter-valley electron backscattering ( $\delta k \simeq 2 k_{F}$ ) in sufficiently long nanotubes can be described by a Luttinger liquid model (for a short range electron-electron interaction) or a Wigner crystal model (for an unscreened Coulomb interaction). The evaluation of persistent currents in these model can be found in $[38,39]$ (see also the review [16]). In all cases mentioned the nanotube chirality did not influence the persistent currents at all. In this section we consider long-range («soft») defects which can induce only intra-valley electron scattering. For these processes chiral effects are significant and they will determine the properties of the persistent current.

The Hamiltonian of the nanotube in our model is $H=H_{j}+\hat{V}_{s}(x)$ where $H_{ \pm}$and $\hat{V}_{S}(x)$ are determined by Eqs. (1), (2) and we will model the spatial dependence of the scattering potential by a rectangular barrier in the local limit (see the previous section). By placing the scatterer at some specific point $(x=a)$ we have two sets of planewave solutions of the Dirac equation, one to the left $(l)$ and one to the right $(r)$ of the scatterer. For the «t-valley» they are as follows $(j=l, r)$

$$
\Psi_{j}=\left(\begin{array}{l}
\exp \left(i k_{F} x\right)\left[A_{j} \exp (i k x)+B_{j} \exp (-i k x)\right] \\
\exp \left[i\left(k_{F} x-\theta\right)\right]\left[A_{j} \exp (i k x)-B_{j} \exp (-i k x)\right]
\end{array}\right) .
$$

The coefficients $A_{j}, B_{j}$ are found from two pairs of equations. The first pair, 


$$
\Psi_{r}(x+L)=\exp \left(2 \pi i \frac{\Phi}{\Phi_{0}}\right) \Psi_{l}(x)
$$

represents the Aharonov-Bohm boundary condition ( $L$ is the ring circumference, $\Phi_{0}=h c / e$ is the flux quantum, and we note that in the absence of a scatterer $\Psi_{l}(x) \equiv \Psi_{r}(x)$ so that in this case Eq. (10) is the familiar twisted boundary condition for a particle on a ring threaded by a magnetic field). The second pair of equations gives the connection between the amplitudes $\Psi_{l}(a)$ and $\Psi_{r}(a)$ induced by local potential scattering. It can be represented in the form (see the previous section)

$$
\left(\begin{array}{c}
A_{l} \\
B_{l}
\end{array}\right)=\left(\begin{array}{cc}
\frac{1}{t} & \frac{r}{t} \\
\frac{r}{t} & \frac{\exp \left(i 2 \pi \phi_{c}\right)}{t^{*}}
\end{array}\right)\left(\begin{array}{l}
A_{r} \\
B_{r}
\end{array}\right)
$$

where $t, r$ are the transmission and reflection amplitudes defined in Eq. (4) and $\phi_{c}=\left(U_{0} / 2 \pi\right) \cos \theta$ is the chiral flux. Notice that the matrix $\hat{A}$ in Eq. (11) is not a transfer matrix (in particular $\operatorname{det} \hat{A}=\exp \left(i 2 U_{0} \cos \theta\right) \neq 1$ for $\left.U_{0} \neq 0\right)$. In our case the scattering is a two-channel process (we have an additional spinor index) and the corresponding transfer matrix is $4 \times 4$-matrix. It is easy to check that the Dirac current is conserved in the scattering process. An analogous set of equations describes the scattering of electrons in the $-k_{F}$-valley.

The solvability condition for the above linear equations results in the spectral equation

$$
\sqrt{D(\theta)} \cos \left(2 \pi \frac{\Phi}{\Phi_{0}} \mp k_{F} L \pm U_{0} \cos \theta\right)=\cos \left[k L-\delta_{b}(\theta)\right]
$$

where $D(\theta), \delta_{b}(\theta)=\delta_{f}(\theta)-U_{0} \cos \theta$ are determined by Eqs. (5), (6). Here the upper (lower) signs correspond to the energy spectrum in the $+k_{F}$-valley ( $-k_{F}$-valley). The term $k_{F} L$ results in a statistical flux («parity effects» [40]) in the persistent current of an isolated ring (where the total number of particles is fixed). Chiral tunneling introduces an additional term $\pm U_{0} \cos \theta$, which we named the «chiral phase» $\varphi_{c}$ (or chiral flux $\phi_{c}=\varphi_{c} / 2 \pi$ ). Notice that particles in $( \pm)$-valleys feel chiral fluxes of opposite signs (the 1.h.s. of Eq. (12).

In the limiting case of a local scatterer that we are interested in, neither the transmission probability nor the scattering phases depend on energy. So the energy spectrum $\left(E= \pm \hbar v_{F} k\right)$ is

$$
\begin{gathered}
E_{n, j}=\frac{\hbar v_{F}}{L}\left\{ \pm \arccos \left[\sqrt{D(\theta)} \cos \left(2 \pi \frac{\Phi}{\Phi_{0}}-j \varphi_{\mathrm{eff}}\right)\right]+\right. \\
\left.+\delta_{b}(\theta)+2 \pi n\right\}
\end{gathered}
$$

where $n=0, \pm 1, \pm 2, \ldots, j= \pm$ and $\varphi_{\text {eff }}=k_{F} L-U_{0} \cos \theta$ is the effective dimensionless flux. The evaluation of the persistent current for a ring at given chemical potential $\mu$,

$$
J(\Phi ; \theta)=-c \frac{\partial \Omega}{\partial \Phi}
$$

(where $\Omega$ is the grand canonical thermodynamic potential) for the spectrum given by Eq. (13) is straightforward. The result at finite temperatures $T$ is (we consider here spinless electrons)

$$
\begin{aligned}
& J=\frac{2}{\pi} I_{0} \frac{T}{T_{*}} \sum_{j= \pm ; k=1}^{\infty} \frac{\sin \left(2 \pi \frac{\Phi}{\Phi_{0}}+j \varphi_{\mathrm{eff}}\right)}{\sqrt{D^{-1}(\theta)-\cos ^{2}\left(2 \pi \frac{\Phi}{\Phi_{0}}+j \varphi_{\mathrm{eff}}\right)}} \times \\
& \times \frac{\sin \left\{k \arccos \left[\sqrt{D(\theta)} \cos \left(2 \pi \frac{\Phi}{\Phi_{0}}+j \varphi_{\mathrm{eff}}\right)\right]\right\} \cos k \delta(\mu, \theta)}{\sinh \left(k \frac{T}{T_{*}}\right)} .
\end{aligned}
$$

Here $I_{0}=e v_{F} / L, T_{*}=\hbar v_{F} / \pi L, \delta(\mu, \theta)=\mu L / \hbar v_{F}-\delta_{b}(\theta)$. We see from Eq. (15) that there is a spontaneous persistent current (i.e., at zero external magnetic flux $\Phi=0$, see the discussion in the review [17]) in each valley. However, at equilibrium and for a ring with a fixed chemical potential, for which the energy levels in the two valleys are equally populated, the net persistent current at zero flux vanishes, $J(\Phi=0 ; \theta)=0$. This conclusion is of course a consequence of the time-reversal invariance of our problem in the absence of an external magnetic field.

The influence of temperature on the persistent current in SWNTs is standard - at hight temperatures $\left(T \gg T_{*}\right)$ the amplitude of Aharonov-Bohm oscillations is exponentially small. The crossover temperature $T_{*}$ is determined by the level spacing. In what follows we will consider the low temperature limit $T \ll T_{*}$ and the case of zero chemical potential, which corresponds to undoped nanotubes. The most interesting situation is when there is resonant chiral tunneling $\left(D=1, \delta_{b}^{\text {res }}=0\right)$. In this case the formula for the persistent current takes the form

$$
J_{\text {res }}=\frac{8}{\pi} \frac{e v_{F}}{L} \sum_{k=1}^{\infty} \frac{\sin \left(2 \pi k \frac{\Phi}{\Phi_{0}}\right)}{k} \cos \left[k \pi\left(\frac{N}{2}-n_{0}\right)\right],
$$

where $N$ is the total number of spin-1/2 electrons in the ring (in the half-filled conduction band) and $n_{0}=U_{0} \cos \theta / \pi$. As readily seen from Eq. (16), the current at $\Phi \neq 0$ persists even at the Dirac point $(\mu=0)$. In an undoped SWNT ring $(\mu=0)$ the total number of particles with energy $E \leq 0$ and momentum $-k_{F}<k<+k_{F}$ is $N=4(m+2)(m$ is an integer). A degeneracy factor of 4 comes from $\mathrm{spin} \times$ helicity degeneracy and another factor 


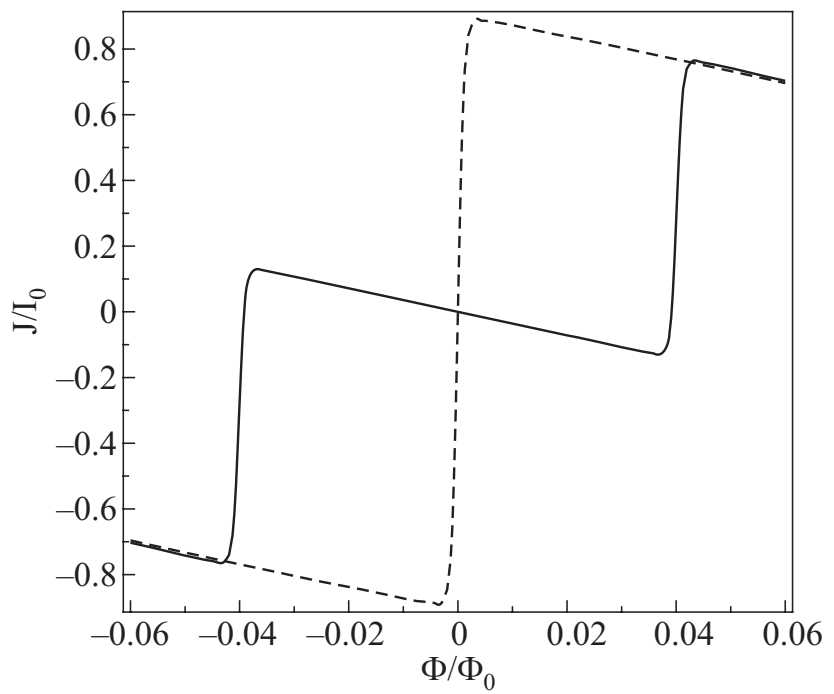

Fig. 2. Persistent current as a function of magnetic flux for different values of the chiral angle near the off-resonance point $\left(\varphi_{c}=\pi\left(n_{0}+1 / 2\right) ; \theta=0.402 \mathrm{rad}, U_{0}=70\right.$ results in $n_{0}=20$; this integer numerates the 20th off-resonance point counted from $\theta=\pi / 2$ in $D(\theta)$ dependence): the solid curve corresponds to $\theta=0.398 \mathrm{rad}$, the dashed curve to $\theta=0.418 \mathrm{rad}$.

of 2 comes from the double degeneracy of zero-energy modes at each Dirac point. We see that in the absence of chiral tunneling $\left(U_{0}=0\right)$ the persistent current is always paramagnetic (see, e.g., [37]). Now the response of the ring to a magnetic field depends on the parity of the chiral resonance: for even $n_{0}$ the persistent current is paramagnetic, for odd $n_{0}$ we have a diamagnetic persistent current.

At low temperatures, $T \rightarrow 0$, and for $\mu=0$ in the offresonance case $\left(U_{0} \cos \theta \rightarrow \pi\left(n_{0}+1 / 2\right)\right)$ the persistent current even for a zigzag nanotube (maximal backscattering coefficient $R=1 / 4$ ) is highly non-harmonic (it has a prominent sawtooth-like shape). Depending on the parity of $n_{0}$ and the approach to the off-resonance point, the current is either paramagnetic (even $n_{0}$, from the «left» of the off-resonance point) or diamagnetic (even $n_{0}$, from the «right» of the off-resonance point) and vice versa (see Fig. 2). The change from a para- to a diamagnetic response is associated with a jump of the backscattering phase Eq. (6) by $\Delta \delta_{b}=\pi$ each time one passes through the offresonance condition. So the parity of the resonance $\left(n_{0}\right)$ determines the type of the response up to the off-resonance point, where it is «smoothly» changed (see Fig. 2).

In an isolated ring (with a fixed total number of particles) at $T=0$ the population of zero-energy modes can be asymmetric. Then the ring will support a spontaneous persistent current (the sign of current — clockwise or counter-clockwise - will be determined by the concrete choice of zero-mode population by chiral electrons). These currents are not equilibrium currents [41] in the presence of even small $2 k_{F}$-backscattering, which tends to symmetrize the population of the zero-energy modes.

\section{Chiral effects on the Josephson current}

We consider the influence of nanotube chirality on the supercurrent through an SNS junction based on a singlewall carbon nanotube. The standard approach for describing $\mathrm{S} / \mathrm{SWNT} / \mathrm{S}$ junctions is to model the normal region as a Luttinger liquid (see, e.g., the review [42]). In Luttinger liquids there are specific phenomena (strong enhancement of backscattering off local impurities, spin-charge and charge-entropy separation) which are absent in Fermi liquids. The most important property for charge transport is the strong renormalization of the scattering potential by electron-electron interactions, which results in a power-low dependence of the differential conductance on temperature and bias voltage (the Kane-Fisher effect [54]). Backscattering processes $\left(\delta k \simeq 2 k_{F}\right)$ mix quasiparticles (electrons and holes) of different helicities and chiral properties of the nanotube cease to be relevant. It is known [44] that in a fully transparent junction (without normal backscattering) the Josephson current is not renormalized by electronelectron interactions. In long SNS tunnel junctions the influence of interactions results mostly in a renormalization (suppression for repulsive interactions) of the junction transparency [45] (see also the review [32] and references therein). In both cases the chirality of the junction does not influence the supercurrent at all.

There is a certain analogy between supercurrents in long SNS junctions ( $d \gg \xi_{0}$, where $d$ is the length of the junction and $\xi_{0}=\hbar v_{F} / \Delta_{0}$ is the superconducting coherence length) and persistent currents in normal-metal ballistic rings (see, e.g., [46]). We have seen already that chiral tunneling leads to new effects in persistent currents. What is the effect of chiral tunneling on the Josephson current through an S/SWNT/S junction?

To calculate the supercurrent in an SNS junction from the expression

$$
J=\frac{4 e}{\hbar} \frac{\partial \Omega}{\partial \varphi}
$$

(where $\Omega$ is the thermodynamic potential, $\varphi$ is the phase difference and the factor 4 counts spin and pseudospin degeneracies) we need to know the spectrum of Andreev bound states in the normal region (here a SWNT containing a «soft» scatterer). Although we know the scattering characteristics of our potential from Eqs. (5), (6), we can not from the very beginning use the known formulae for the spectrum of Andreev-Kulik levels [47] and the Josepson current in an SNS junction in terms of junction transparency. The Andreev scattering in graphene was shown [48] to exhibit peculiarities (specular Andreev reflection) as compared to the ordinary SNS junctions. Therefore we will follow the standard approach and find the spectrum by 
solving the Bogoliubov-de Gennes (BdG) equation with the order parameter $\Delta(x)$ constant in the superconductors $\left(\Delta(x)=\Delta_{0} \mathrm{e}^{i \phi_{L}}\right.$ for $x<0, \Delta(x)=\Delta_{0} \mathrm{e}^{i \phi_{R}}$ for $x>d$, here $\left.\varphi_{L}-\varphi_{R}=\varphi\right)$ and $\Delta(x)=0$ in the normal region. The scatterer is placed at the point $x=l$ inside the normal region.

By matching the plane wave solutions of the BdG equation in the normal region at points $x=0, l, d$ we derive the equation for the bound state energies (Andreev-Kulik levels [47])

$$
\begin{gathered}
\cos \left[\frac{2 E d}{\hbar v_{F}}-2 \arccos \frac{E}{\Delta_{0}}\right]= \\
=D(\theta) \cos \varphi+R(\theta) \cos \left[\frac{2 E(2 l-d)}{\hbar v_{F}}\right],
\end{gathered}
$$

where $R(\theta)$ and $D(\theta)$ are defined in Eq. (5). The spectral equation, Eq. (18), is the standard equation for the bound state energies in an SNINS junction (I denotes the scatterer inside the normal region). The phase factor which an electron (hole) picks up in the process of chiral tunneling is energy independent in our model. The phases acquired by electrons and holes are opposite in sign and cancel out in the spectral equation. Besides, contrary to the problem of persistent currents in a ballistic ring, the effective flux (both statistical and chiral) $\varphi_{\text {eff }}=k_{F} L-U_{0} \cos \theta$ does not enter the spectral equation (18). In the process of Andreev reflection at an $\mathrm{S} / \mathrm{N}$ boundary two electrons with small total momentum $\left(k \ll k_{F}\right)$ penetrate into the bulk superconductor. It means that an electron in the $+k_{F}$-valley is reflected as a hole in the $-k_{F}$-valley (and vice versa). The electron and the hole have opposite momenta and opposite pseudospin (but the same helicity). The two possible helicities $( \pm 1)$ result in an additional factor 2 in the definition of the Josephson current Eq. (17). We have already seen in the previous section that particles in different valleys carry effective fluxes $\varphi_{\text {eff }}$ with opposite signs. These contributions to the spectral equation, Eq. (17), cancel out. As a result all information in Eq. (17) specific to SWNTs is hidden in the transmission probability $D(\theta)$. In particular, the nanotube chirality does not influence the Josephson current at all in the absence of normal scattering $\left(U_{0}=0\right)$.

What are then the effects of chiral tunneling on the Josephson current? In chiral nanotubes the junction transparency is an oscillating function of the strength, $U_{0}$, of the «soft» scattering potential. Therefore one can expect an anomalous (non-monotonic) behavior of the critical current as a function of potential strength. For resonant chiral tunneling, $U_{0} \cos \theta=\pi n_{0}$, the junction becomes fully transparent $\left(D_{r}=1\right)$ and the supercurrent through an SNINS junction coincides with (i) the Josephson current through a superconducting constriction (for a short junction $d \ll \xi_{0}, \quad \xi_{0}$ is the superconducting coherence length) [49] $J_{\max }^{(s)}(\varphi)=(2 e \Delta / \hbar) \sin (\varphi / 2)$ (the additional factor 2 in this formula is due to pseudospin degeneracy) or (ii) the supercurrent through a long $d \gg \xi_{0}$ transparent junction $J_{\max }^{(l)}(\varphi)=2\left(e v_{F} / d\right)(\varphi / \pi)$, where $|\varphi| \leq \pi$. Notice that for resonant chiral tunneling the supercurrent does not depend on the position of the scatterer inside the normal region. For off-resonant tunneling the current in a long junction does depend on the actual position $(x=l)$ of the local scatterer. However, the effect is numerically small. For the two limiting cases of a symmetric $(l=d / 2)$ and a maximally asymmetric $(l=0, d)$ junction the supercurrents are

$$
\begin{aligned}
J_{s}^{\text {off }}(\varphi ; \theta) & =\frac{2}{\pi} \frac{e v_{F}}{d} \frac{\cos ^{2} \theta \sin \varphi}{\sqrt{1-\left(\sin ^{2} \theta+\cos ^{2} \theta \cos \varphi\right)^{2}}} \times \\
\times & \arccos \left(\sin ^{2} \theta+\cos ^{2} \theta \cos \varphi\right), \\
J_{a}^{\text {off }}(\varphi ; \theta)= & \frac{2}{\pi} \frac{e v_{F}}{d} \frac{\cos ^{2} \theta \sin \varphi}{\sqrt{\left(1+\sin ^{2} \theta\right)^{2}-\cos ^{4} \theta \cos ^{2} \varphi}} \times \\
& \times \arccos \left(\frac{\cos ^{2} \theta \cos \varphi}{1+\sin ^{2} \theta}\right) .
\end{aligned}
$$

The behavior of the supercurrents $J_{s, a}$ for different chiral angles is shown in Fig. 3. We see that at given $\theta$ and $\varphi$ the current through a symmetric junction is always larger (although the effect is numerically small) than the current through an asymmetric junction.

There are proposals to use the pair of Andreev levels in a short SNS junction as a qubit («Andreev qubit», see Ref. 50) and for cooling of nanoelectromechanical devices [51]. In these proposals the coherent dynamics of the Andreev-Kulik levels occur deep inside the gap region ( $E \ll \Delta_{0}, \Delta_{0}$ is the superconducting order parameter).

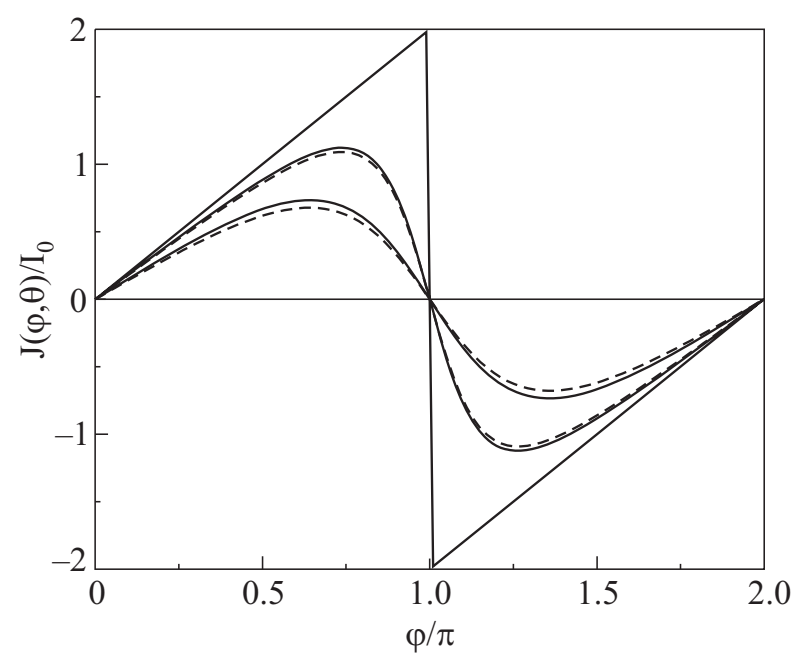

Fig. 3. The Josephson current(in units $I_{0}=e v_{F} / d$ ) in a long junction, $U_{0}=70$; solid curve corresponds to the chiral resonance ( $\theta=0.158 \mathrm{rad})$ and two off-resonance cases $\theta=0.276 \mathrm{rad}$ and $\theta=0.507 \mathrm{rad}$ (symmetric junction); the corresponding currents in asymmetric junction are represented by the dashed curves. 
This regime could be reached only for almost fully transparent junctions and chiral nanotube-based junctions could be promising candidates for the fabrication of SNS junctions with high transparency $D-1 \ll 1$.

In the general case of non-resonant chiral tunneling the minimum energy separation between the pair of AndreevKulik levels in a short SNS junction is $E_{g}(\theta)=$ $=2 \Delta_{0} \sqrt{1-D(\theta)}$. The minimal junction transparency $D_{\text {min }}=\cos ^{2} \theta$ is reached at $U_{0} \cos \theta=\pi\left(n_{0}+1 / 2\right)$ (chiral off-resonance). So the gap

$$
E_{g}(\theta)=2 \Delta_{0} \sin \theta
$$

could be arbitrary small for nanotubes with small chiral angles.

\section{Resonant tunneling through a chiral quantum dot}

Resonant electron tunneling in quantum wires (see, e.g., the review [52]) is a coherent quantum mechanical phenomenon which is extremely sensitive to the electron energy spectrum. In transport experiments with quantum dots in the regime of resonant tunneling (resonant transport spectroscopy) one gets valuable information about the electron energies and electron wave functions in the dot by measuring the position and the shape of resonance conductance peaks as a function of the gate voltage. Single-wall carbon nanotubes from the very beginning of their discovery were considered as promising elements for future nanoelectronics. In particular, carbon nanotube-based single electron transistors (SET) were fabricated and electron transport through these molecular devices was studied in a wide range of temperatures (see, e.g., the review [53] and references therein). The observation at low temperatures of Coulomb blockade oscillations and resonant electron tunneling in long (a few hundreds nanometers ) metallic SWNTs means that electrons are delocalized along the whole length of the structure. This fact is usually explained by the specifics (Klein paradox) of scattering of charge carriers (massless Dirac electrons) in SWNTs by longrange tube defects [26]. Chiral tunneling in this sense has already been indirectly observed in SWNT-based quantum dots. Here we consider the direct influence of chiral tunneling on the resonant transport properties of quantum dots.

We will model a «chiral quantum dot» by a finite length $(L)$ metallic chiral SWNT, Eq. (1), with the «soft» local scatterer, Eq. (4) placed at a distance $l$ from the left end of the nanotube. The nanotube is connected to the leads by normal tunnel barriers which results in a finite (small) width $\Gamma$ of the electron energy levels (we will assume the widths to be energy independent). The electron energy spectrum, to lowest order on $\Gamma$, can be found by assuming the end-barriers to be infinite. The corresponding boundary conditions can be formulated as the absence of any electron (Dirac) current through the ends of the nanotube, $j_{D}(x=0, L)=0$. Since scattering at the ends connects electrons in the $+k_{F}$ and $-k_{F}$ valleys the current should be expressed in terms of the 4-spinors $\Psi^{T}=\left(\psi_{+}^{T}, \psi_{-}^{T}\right)$, where $T$ denotes transposition. For our Hamiltonian, Eq. (1), the current looks like

$$
j_{D}(x)=v_{F} \Psi^{\dagger}(x)\left(\begin{array}{cccc}
0 & \mathrm{e}^{i \theta} & 0 & 0 \\
\mathrm{e}^{-i \theta} & 0 & 0 & 0 \\
0 & 0 & 0 & -\mathrm{e}^{-i \theta} \\
0 & 0 & -\mathrm{e}^{i \theta} & 0
\end{array}\right) \Psi(x)
$$

The physically evident solution of the above discussed boundary conditions is the scattering at the boundaries, when a left-moving fermion in the «t»-valley is transformed into a right-moving fermion in the «-»-valley and all analogous processes ( $L^{ \pm} \leftrightarrow R^{\mp}$ ). In a general case this scattering is accompanied by an energy-independent phase shift.

By matching the plane wave solutions of the Dirac equation at the points $x=0, l, L$ using our boundary conditions and the matrix $\mathrm{Eq},(11)$ for $\langle+\rangle-$ and «-»-valleys $(\theta \rightarrow-\theta)$ we derive the following spectral equation

$$
\begin{gathered}
\cos [2 k L-\zeta(\theta)]=R(\theta) \cos [2 k(L-2 l)]+ \\
+D(\theta) \cos \left(2 k_{F} L-2 U_{0} \cos \theta\right),
\end{gathered}
$$

where the total scattering phase $\zeta(\theta)$ is

$$
\begin{gathered}
\zeta(\theta)=\arctan \left[\frac{\sin \left(2 U_{0} \cos \theta\right) \cos \theta}{\cos ^{2}\left(U_{0} \cos \theta\right) \cos ^{2} \theta-\sin ^{2}\left(U_{0} \cos \theta\right)}\right]= \\
=2\left[\delta_{f}(\theta)-U_{0} \cos \theta\right] .
\end{gathered}
$$

Notice that Eq. (23) coincides with Eq. (18) of Ref. 54. However, the expressions for the scattering data in our case are different (the assumption for the scattering potential for which the transmission and reflection amplitudes were obtained in [54] is not satisfied in our model). The derived spectral equation has a simple physical interpretation. The phase terms in Eq. (23) depend on: (i) the «quantization length» $2 L$ (at the boundaries inter-valley electron scattering occurs and one needs a $2 L$-path to form a closed trajectory), (ii) the double forward scattering phase $2 \delta_{f}(\theta)$ which the particle picks up by passing the scattering potential twice, and (iii) the double chiral phase $2 U_{0} \cos \theta=2 \varphi_{c}$, which is added to the geometrical phase $2 k_{F} L$.

Since the momentum $k_{F}$ is defined for an undoped nanotube it can be expressed through the total number of particles in a half-filled conduction band as $k_{F} L=\pi N / 2$, where $N=4(m+2), m$ is an integer; see the discussion in Sec. 3). We see that the phase associated with the terms $k_{F} L$ does not influence the spectral properties of chiral quantum dots. For armchair nanotubes $(R(0)=0$ and $\delta_{f}(0)=2 U_{0}+\pi n$ on module $\left.2 \pi\right)$ the spectral equation reads $\cos \left(2 k L-2 U_{0}\right)= \pm \cos 2 U_{0}$, which results in two 
sets of equidistant energy levels $\left(\Delta E=\pi \hbar v_{F} / L\right)$ which are shifted relatively each other by $\delta_{f}(0) \hbar v_{F} / L$. Notice that for $\delta_{f} \simeq \pi$ the level spacing is approximately halved (this halved level spacing is usually considered as an averaged level spacing in SWNT). In the case of chiral resonant tunneling the electron energy spectrum in a chiral nanotube does not depend of the position of the chiral scatterer (as for armchair nanotubes). It is still equidistant with energy spacing $\pi \hbar v_{F} / L$. In the general case of nonresonant scattering the energy spectrum is quasi-random for irrational values $l / L$. The distribution of the number of energy levels on the level spacing (normalized by $\left.\Delta E=\pi \hbar v_{F} / L\right)$ for a given potential strength and different chiral angles is shown in Fig. 4. The levels, which differ in energies by less then the level width $\Gamma$, are considered as «degenerate». We see that two $\delta$-function-like peaks for $\theta=0$ are developed for sufficiently strong backscattering ( $D \ll 1$ ) to the distribution which resembles WignerDyson distribution. Although in our model (real potentials $V_{d}, V_{o}$ of equal strengths) small transmission coefficients correspond to nonphysical chiral angles ( $\theta$ close to $\pi / 2$ ) small transparencies in chiral tunneling could be realized
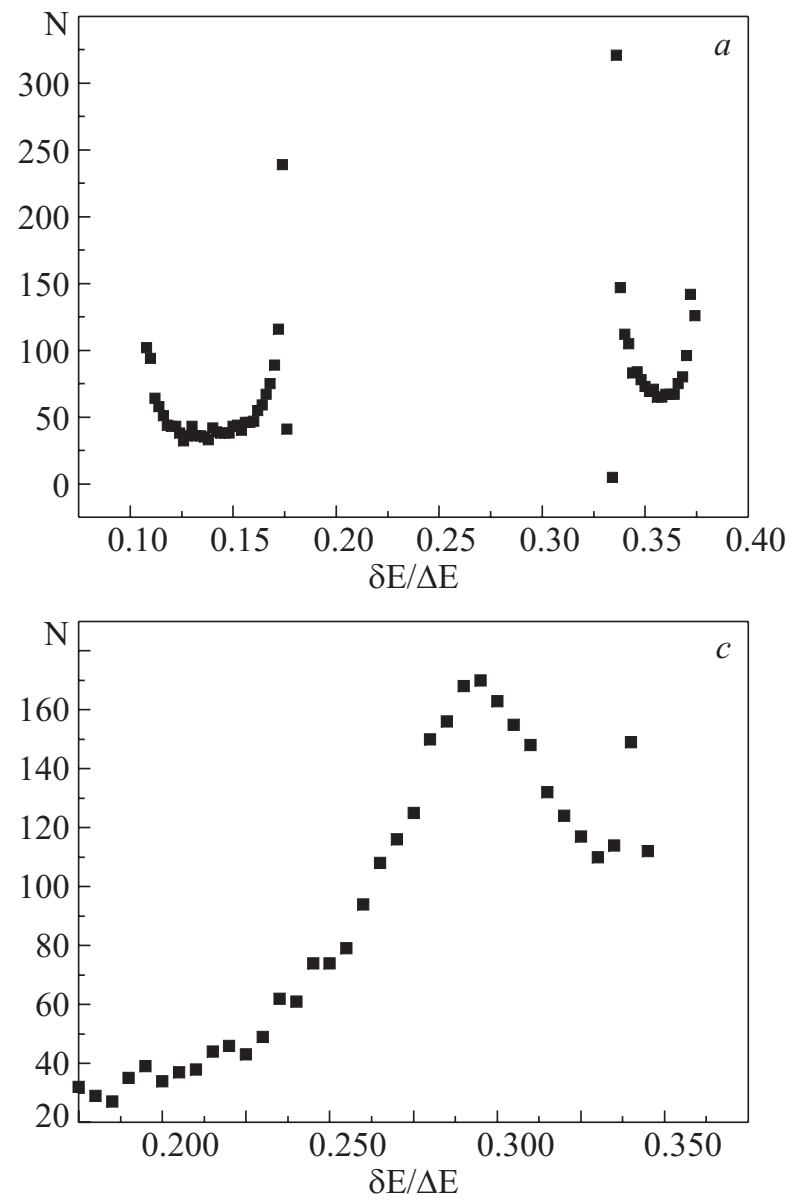

in the general case of nonsymmetric potentials.

In the regime of resonant electron tunneling through quantum dots the distribution of spacings of the peak (maximum) conductances as a function of gate voltages is determined by the distribution of the level spacings for electron energy spectrum. We showed that the mechanism of chiral tunneling is sensitive to the chirality of nanotubes. Therefore this phenomenon could be used for determination of nanotube chiral angle in resonant transport spectroscopy.

\section{Conclusion}

In this paper we have introduced the new concept of chiral tunneling in metallic single-wall carbon nanotubes. There are significant differences between the Klein (or chiral) tunneling of massless 2D Dirac-like particles in graphene (well studied in recent years; see the review [27]) and the chiral tunneling of 1D massless fermions in SWNTs. In the 2D scattering problem in graphene even a scalar electromagnetic potential can backscatter massless Dirac electrons if the incident angle of scattering particle is not close to zero (for normal incidence the transmission
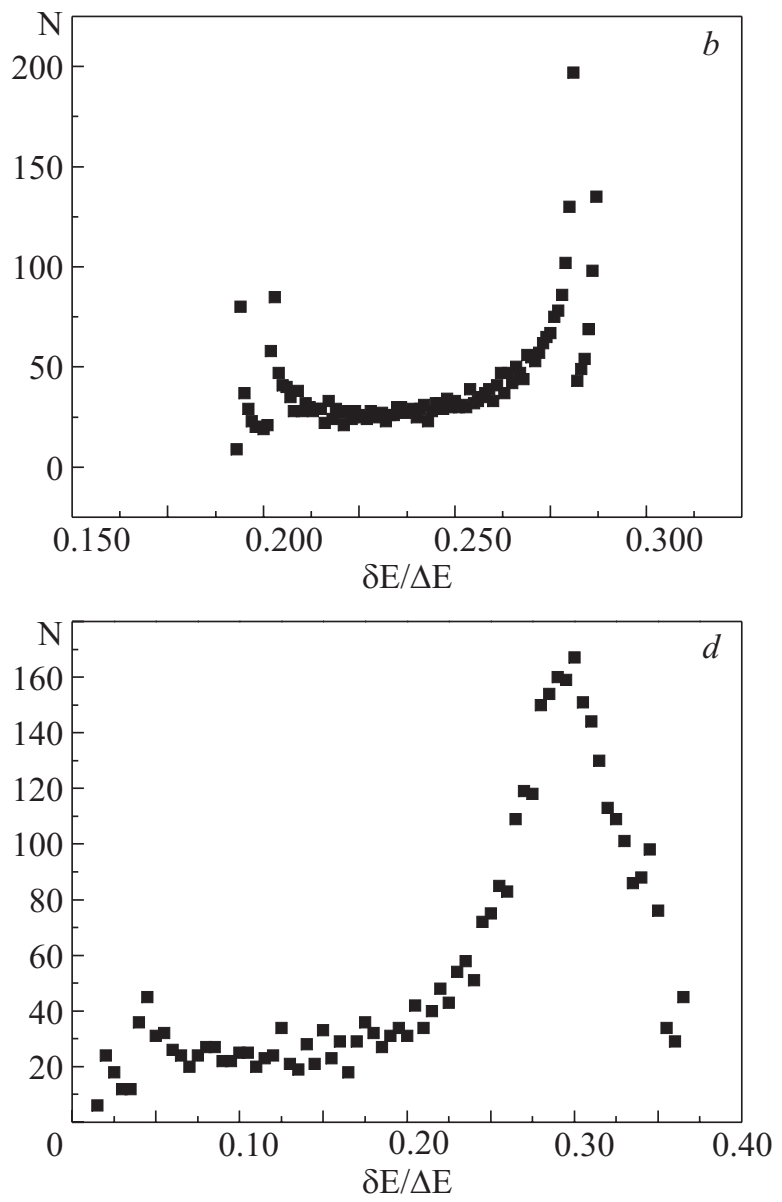

Fig. 4. The distribution of the number of energy levels on the level spacing (normalized by $\Delta E=\pi \hbar v_{F} / L$ ) for $U_{0}=20$ and different chiral angles: to $\theta=0.49 \mathrm{rad}(D=0.8)(a), \theta=0.52 \mathrm{rad}(D=0.75)(b), \theta=0.955 \mathrm{rad}(D=0.4)(c), \theta \approx \pi / 2(D=0.06)(d)$. For the armchair nanotube we have two sets of equidistant energy levels: $\delta E / \Delta E=0.13$ and 0.36 . The distribution is shown for $l / L=1 / 2 \pi, \Gamma=0.002$. 
coefficient is always $D=1$ ). In a SWNT the scattering is a 1D problem and one can expect finite-probability reflection of massless Dirac particles only in chiral nanotubes where spiral-like electron «motion» along the cylinder axis mimics some features of 2D scattering. Besides, in nontrivial scattering $(R \neq 0)$ the scattering potential can not be purely electrostatic («diagonal» in our representation of Dirac matrices). The presence of non-diagonal components, which are induced by a pseudovector potential (pseudomagnetic effects), are crucial in chiral tunneling in nanotubes. In our consideration we use a phenomenological approach to the problem and postulate the form of matrix scattering potential Eq. (2) in order to study the general properties of chiral tunneling. We know at least one example - the effective scattering potential induced by fullerene molecules in nano-peapods, when such a matrix potential was derived microscopically [28]. Notice that magnetic potentials in nanostructures are as a rule longrange and they are consistent with our assumption of a «smooth» scattering potential.

We showed that in chiral tunneling not only is a (small) reflection probability an important physical characteristic but the chiral phase, $\varphi_{c}=U_{0} \cos \theta$, the quantity associated with the effective vector potential experienced by the particle in the process of tunneling, plays a significant role as well. It is worth to note that the forward and backward scattering phases in chiral tunneling are related by the simple expression $\delta_{f}-\delta_{b}=\varphi_{c}$. The quantized chiral phase determines the conditions for resonant $\left(\varphi_{c}=\pi n, n\right.$ is the integer, $\left.D_{r}=1\right)$ and off-resonant $\left(\varphi_{c}=\pi(n+1 / 2)\right.$, $D_{o}=\cos ^{2} \theta$ ) chiral tunneling.

The chiral phase is added to the magnetic flux in the problem of Aharonov-Bohm oscillations and its appearance can result in a spontaneous persistent current in a ring with an asymmetric population of zero-energy modes. Since the particles with opposite helicities acquire chiral phases of opposite signs, the chiral phases are cancelled in the Josephson current problem when a pair of electrons $\left(-k_{F},+k_{F}\right)$ tunnel to the bulk superconductor. We demonstrated the nontrivial role the chiral phase plays in various phase coherent phenomena in nanostructures.

The last question we would like to discuss here is the influence of electron-electron interactions on chiral tunneling. We will assume that the interaction is not strong, otherwise the Luttinger liquid effects which strongly enhance $2 k_{F}$-backscattering violate our assumption of a smooth diagonal scattering potential. It is physically evident that for the conditions of resonant chiral tunneling $\left(D_{r}=1\right)$ electron-electron interactions do not renormalize chiral scattering potentials at all. Off-resonance there is finite backscattering and one could expect its enhancement by (repulsive) interaction effects. This problem needs special consideration. We briefly mention here that in the limit of weak interactions, when the renormalization is induced by electron scattering on Friedel oscillations [55], the naive mean-field approximation of the interaction potential does not lead to logarithmic infra-red singularities in the backscattering amplitudes.

We gratefully acknowledge discussions with L. Gorelik, A. Kadigrobov, S. Kulinich and V. Shumeiko. AVP thanks V.Yu. Monarkha for help with numerical calculations. This work was supported in parts by the Swedish VR, the European Commission (FP7-ICT-2007-C; proj No 225955 STELE), and the Korean WCU programme funded by MEST through KOSEF (R31-2008-000-10057-0). The research of ENB and UL was supported by the US Department of Energy under Grant No. FG05-86ER45234. IVK acknowledges the Department of Physics at the University of Gothenburg for hospitality.

1. Y. Aharonov and D. Bohm, Phys. Rev. 115, 485 (1959).

2. R.G. Chambers, Phys. Rev. Lett. 5, 3 (1960).

3. S. Olariu and I.I. Popescu, Rev. Mod. Phys. 57, 339 (1985).

4. I.O. Kulik, Pisma Zh. Eksp. Teor. Fiz. 11, 407 (1970) [JETP Lett. 11, 275 (1970)].

5. L. Gunther and Y. Imry, Solid State Commun. 7, 1391 (1969).

6. F. Bloch, Phys. Rev. B2, 109 (1970).

7. N.B. Brandt, D.V. Gitsu, A.A. Nikolaeva, and Ya.G. Ponomarev, Pisma Zh. Eksp. Teor. Fiz. 24, 304 (1976) [JETP Letters 24, 272 (1976)].

8. N.B. Brandt, E.N. Bogachek, D.V. Gitsu, G.A. Gogadze, I.O. Kulik, A.A. Nikolaeva, and Ya.G. Ponomarev, Fiz. Nizk. Temp. 8, 718 (1982) [Sov. J. Low Temp. Phys. 8, 358 (1982)].

9. E.N. Bogachek and G.A. Gogadze, Zh. Eksp. Teor. Fiz. 63, 1839 (1972) [Sov. Phys. JETP 36, 973 (1973)].

10. E.N. Bogachek, A.G. Scherbakov, and U. Landman, Phys. Rev. B63, 115323 (2001).

11. V. Chandrasekhar, R.A. Webb, M.J. Brady, M.B. Ketchen, W.J. Gallagher, and A. Kleinsasser, Phys. Rev. Lett. 67, 3578 (1991).

12. M. Büttiker, Y. Imry, and R. Landauer, Phys. Lett. A96, 365 (1983).

13. D. Mally, C. Chapelier, and A. Benoit, Phys. Rev. Lett. 70, 2020 (1993).

14. Y. Imry, Introduction to Mesoscopic Physics, Oxford Univ. Press (1997).

15. I.V. Krive and A.S. Rozhavsky, Int. J. Mod. Phys. B6, 1255 (1992).

16. A.A. Zvyagin and I.V. Krive, Fiz. Nizk. Temp. 21, 687 (1995) [Low Temp. Phys. 21, 533 (1995)].

17. I.O. Kulik, Fiz. Nizk. Temp. 30, 705 (2004) [Low Temp. Phys. 30, 528 (2004)].

18. Y.I. Latyshev, O. Laborde, P. Monceau, and S. Klaumunzer, Phys. Rev. Lett. 78, 919 (1997).

19. E.N. Bogachek, I.V. Krive, I.O. Kulik, and A.S. Rozhavsky, Phys. Rev. B42, 7614(1990).

20. E.N. Bogachek, I.V. Krive, I.O. Kulik, and A.S. Rozhavsky, Zh. Eksp. Teor. Fiz. 97, 603 (1990) [Sov. Phys. JETP 70, 336 (1990)]. 
21. I.O. Kulik, A.S. Rozhavskii, and E.N. Bogachek, JETP Lett. 47, 303 (1988).

22. E.N. Bogachek, I.A. Romanovsky, and U. Landman, Phys. Rev. B78, 174515 (2008).

23. J. Liu, H. Dai, J.H. Hafner, D.T. Colbert, R.E. Smalley, S.J. Tans, and C. Dekker, Nature 385, 780 (1997).

24. R. Martel, H.R. Shea, and Ph. Avouris, Nature 398, 299 (1999).

25. A.Yu. Kasumov, R. Deblock, M. Kociak, B. Reulet, H. Bouchiat, I.I. Khodos, Yu.B. Gorbatov, V.T. Volkov, C. Journet, and M. Burghard, Science 284, 1508 (1999).

26. P.L. McEuen, M. Bockrath, D.H. Cobden, Y.G. Yoon, and S.G. Louie, Phys. Rev. Lett. 83, 5098 (1999).

27. A.H.C. Neto, F. Guinea, N.M.R. Peres, K.S. Novoselov, and A.K. Geim, Rev. Mod.Phys. 81, 109 (2009).

28. C.L. Kane, E.J. Mele, A.T. Johnson, D.E. Luzzi, B.W. Smith, D.J. Hornbaker, and A. Yazdani, Phys. Rev. B66, 235423 (2002).

29. R. Saito, G. Dresselhaus, and M.S. Dresselhaus, Physical Properties of Carbon Nanotubes, Imperial College Press, London (1998).

30. Carbon Nanotubes: Synthesis, Structure, Properties, and Applications, M.S. Dresselhaus, G. Dresselhaus, and Ph. Avouris (eds.), Topics Appl. Phys. Vol. 80, Springer (2001).

31. M.I. Katsnelson, K.S. Novoselov, and A.K. Geim, Nature Phys. 2, 620 (2006).

32. I.V. Krive, Fiz. Nizk. Temp. 24, 458 (1998) [Low Temp. Phys. 24, 377 (1998)].

33. I.V. Krive, Phys. Rev. B59, 12338 (1999).

34. M. Büttiker, Y. Imry, and M.Ya. Azbel, Phys. Rev. A30, 1982 (1984).

35. R.C. Haddon, Nature 388, 31 (1997).

36. M.F. Lin and D.S. Chuu, Phys. Rev. B57, 6731 (1998).

37. A.A. Odintsov, W. Smit, and H. Yoshioka, Europhys. Lett. 45, 598 (1999).

38. I.V. Krive, P. Sandstrom, R.I. Shekhter, S.M. Girvin, and M. Jonson, Phys. Rev. B52, 16451 (1995).
39. A.O. Gogolin and N.V. Prokofiev, Phys. Rev. B50, 4921 (1994).

40. D. Loss, Phys. Rev. Lett. 69, 343 (1992).

41. T. Swahn, E.N. Bogacheck, Y.M. Galperin, M. Jonson, and R.I. Shekhter, Phys. Rev. Lett. 73, 162 (1994).

42. I.V. Krive, R.I. Shekhter, and M. Jonson, Fiz. Nizk. Temp. 32, 1171 (2006) [Low Temp. Phys. 32, 887 (2006)].

43. C.L. Kane and M.P.A. Fisher, Phys. Rev. Lett. 68, 1220 (1992).

44. D.L. Maslov, M. Stone, P.M. Goldbart, and D. Loss, Phys. Rev. B53, 1548 (1996).

45. R. Fazio, F.W.J. Hekking, and A.A. Odintsov, Phys. Rev. Lett. 74, 1843 (1995).

46. I.V. Krive, S.I. Kulinich, R.I. Shekhter, and M. Jonson, Fiz. Nizk. Temp. 30, 738 (2004) [Low Temp. Phys. 30, 554 (2004)].

47. I.O. Kulik, Zh. Eksp. Teor. Fiz. 57, 1745 (1969) [Sov. Phys. JETP 30, 944 (1970)].

48. C.W.J. Beenakker, Phys. Rev. Lett. 97, 067007 (2006).

49. I.O. Kulik and A.N. Omelyanchouk, Fiz. Nizk. Temp. 3, 945 (1977) [Sov. J. Low Temp. Phys. 3, 459 (1977)].

50. A. Zazunov, V.S. Shumeiko, E.N. Bratus', J. Lantz, and G. Wendin, Phys. Rev. Lett. 90, 87003 (2003); A. Zazunov, V.S. Shumeiko, E.N. Bratus', and G. Wendin, Phys. Rev. B71, 214505 (2005).

51. G. Sonne, M.E. Peña-Aza, L.Y. Gorelik, R.I. Shekhter, and M. Jonson, Cooling of a Suspended Nanowire by an AC Josephson Current Flow, arXiv: 1002.1207 (2010).

52. I.V. Krive, A. Palevski, R.I. Shekhter, and M. Jonson, Fiz. Nizk. Temp. 36, 155 (2010) [Low Temp. Phys. 36, 119 (2010)].

53. J. Nygård, D.H. Cobden, M. Bockrath, P.L. McEuen, and P.E. Lindelöf, Appl. Phys. A69, 297 (1999).

54. I.V. Krive, R. Ferrone, R.I. Shekhter, M. Jonson, P. Utko, and J. Nygård, New J. Phys. 10, 043043 (2008).

55. K.A. Matveev, D. Yue, and L.I. Glazman, Phys. Rev. Lett. 71, 3351 (1993). 